\title{
Measurement of relaxation between polarization eigenstates in single quantum dots
}

\author{
T. H. Stievater, ${ }^{\text {a) }}$ Xiaoqin Li, T. Cubel, and D. G. Steel ${ }^{\text {b) }}$ \\ The FOCUS Center, Harrison M. Randall Laboratory of Physics, The University of Michigan, \\ Ann Arbor, Michigan 48109-1120 \\ D. Gammon, D. S. Katzer, and D. Park \\ Naval Research Laboratory, Washington, DC 20375-5347
}

(Received 6 August 2002; accepted 9 October 2002)

\begin{abstract}
Low temperature relaxation of excitons between polarization eigenstates in single interface fluctuation quantum dots is studied using copolarized and cross-polarized transient differential transmission spectroscopy. The measured spin relaxation times are on the order of $\sim 100 \mathrm{ps.} \mathrm{Such}$ a spin relaxation time is longer than the reported times for thin quantum wells, but considerably shorter than the predicted times for interface fluctuation quantum dots. (C) 2002 American Institute of Physics. [DOI: 10.1063/1.1526912]
\end{abstract}

In bulk and quantum well semiconductor systems, coupling between excitonic polarization eigenstates can occur following resonant optical excitation, often with time scales faster than the recombination rate. ${ }^{1}$ This coupling, referred to as spin relaxation, can result from phonon interactions, ${ }^{2,3}$ or effects arising from momentum scattering such as D'yakonov-Perel type interactions, ${ }^{4}$ or long-range exciton exchange interactions. ${ }^{5}$ Spin relaxation is expected to be strongly suppressed in quantum dots (QDs) due to the complete discretization of energy eigenstates. Experimental verification of this prediction has become especially important in light of proposals to implement quantum information processing with spin states in $\mathrm{QDs}^{6-9}$ as well as proposals based on the emerging field of spintronics. ${ }^{10}$ Recent results from inhomogeneously broadened ensembles of self-assembled quantum dots have found spin relaxation times to be on the order of $1 \mathrm{~ns}^{2}$ and even longer, ${ }^{11,12}$ consistent with the notion of suppressed spin relaxation in zero-dimensional structures.

In this letter, we measure the spin relaxation rate in a single semiconductor quantum dot formed by interface fluctuations. The measurements are performed using transient differential transmission (DT) spectoscopy on single QD states. This technique has also recently been used to measure biexciton lifetimes ${ }^{13}$ and Rabi oscillations ${ }^{14}$ in single QDs.

The experiments are performed on interface fluctuation QDs formed naturally in a thin $(4.2 \mathrm{~nm})$ GaAs layer grown between two $25 \mathrm{~nm} \mathrm{Al}_{0.3} \mathrm{Ga}_{0.7} \mathrm{As}$ layers. 2 min growth interruptions at each $\mathrm{GaAs} / \mathrm{Al}_{0.3} \mathrm{Ga}_{0.7} \mathrm{As}$ interface lead to the formation of monolayer-high islands which tend to localize the heavy hole excitons in the GaAs layer. ${ }^{15,16}$ Excitons in isolated QDs are probed through $\sim 0.5 \mu \mathrm{m}$ apertures in an aluminum mask laid directly onto the sample's surface. The GaAs substrate is removed to allow for transmission studies and the experiments are performed at $7 \mathrm{~K}$.

The monolayer islands are elongated in the [ $\overline{1} 10]$ direction, leading to linear polarization selection rules for the excitonic transitions, denoted by $\Pi_{x}$ and $\Pi_{y} \cdot{ }^{17} \Pi_{x}$ polarization

\footnotetext{
${ }^{a)}$ Present address: Naval Research Laboratory, Washington, DC 20375.

${ }^{b)}$ Electronic mail: dst@umich.edu; http://www.physics.lsa.umich.edu/dst
}

is defined here to be parallel to the direction of island elongation. The relevant heavy hole excitonic levels for a single asymmetric quantum dot are shown in Fig. 1(a). Relaxation between the two excitonic eigenstates excited by linearly polarized light (denoted $\left|e_{x}\right\rangle$ and $\left|e_{y}\right\rangle$ ) is related to spin relaxation between the states created by circularly polarized light (denoted $\left|e_{-}\right\rangle$and $\left|e_{+}\right\rangle$). In this notation, $\left|e_{x}\right\rangle=1 / \sqrt{2}\left(\left|e_{+}\right\rangle\right.$ $\left.+\left|e_{-}\right\rangle\right),\left|e_{y}\right\rangle=-i / \sqrt{2}\left(\left|e_{+}\right\rangle-\left|e_{-}\right\rangle\right)$, and $\left|e_{+}\right\rangle$is the excitonic state created by $\sigma_{+}$polarization, $\mid m_{J}^{(\text {hole })}=3 / 2$, $\left.m_{J}^{\text {(electron) }}=-1 / 2\right\rangle$, and $\left|e_{-}\right\rangle$is the excitonic state created by $\sigma_{-}$polarization, $\left|m_{J}^{(\text {hole })}=-3 / 2, m_{J}^{\text {(electron) }}=1 / 2\right\rangle$. Since transient DT is not sensitive to single hole or electron spin flips that put the exciton into a dark state, the data and analysis of this letter focus only on the relaxation rates between the two optically active exciton spin states.

Predictions of the spin relaxation times, $1 / \Gamma_{x y}$ or $1 / \Gamma_{y x}$, between the two exciton spin states within the same confinement level in interface fluctuation QDs (intradoublet) are of order nanoseconds. ${ }^{3}$ Single interface fluctuation QD relaxation rates between different confinement levels of opposite polarization have previously been found to be slower than the relaxation rates between states of the same polarization. ${ }^{16}$ These measurements, however, were unable to probe the intradoublet relaxation between the polarization states of the same confinement level.

A synchronously pumped dye laser with pulse width 6 ps is used to perform the pump-probe measurements. The picosecond pulse has sufficient bandwidth $(\sim 300 \mu \mathrm{eV})$ to excite both the $\left|e_{x}\right\rangle$ and $\left|e_{y}\right\rangle$ exciton states of the same doublet (typical splittings are $\sim 20 \mu \mathrm{eV}$ ), as well as sufficiently narrow bandwidth so as not to excite spectrally adjacent QD states (typical splittings are $\sim$ few milli-electron-volts), or biexcitons (which have a binding energy of about 3.5 $\mathrm{meV})^{13,18}$ in the same QD. The biexciton state is neglected in the level diagram of Fig. 1(a), though it is the implicit presence of the biexciton that allows the " $V$ " system to accurately describe the dynamics of exciton relaxation; without this interaction, the two oppositely polarized excitons would behave like uncoupled two-level systems. The pump and probe (delayed by a time $\tau$ ) are focused to a waist at the aperture. Absorption of the probe at the excitonic resonance 


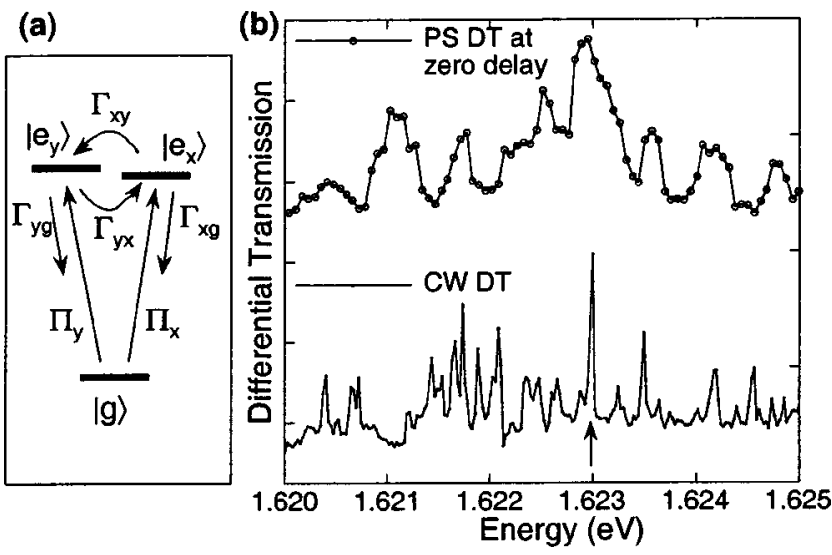

FIG. 1. (a) The three-level system used for the density-matrix analysis of the DT signal. (b) DT spectrum showing single QD states, obtained through a submicron aperture using pulsed (top) and cw (bottom) lasers. The pump and probe are both $\Pi_{y}$ polarized.

creates an induced third order polarization field $\mathbf{P}^{(3)}(t)$. Time-integrated homodyne detection of this polarization field with the transmitted probe represents a DT signal that is proportional to the imaginary part of the amplitude of $\mathbf{P}^{(3)}(t)$. Amplitude modulation at $\sim 1 \mathrm{MHz}$ of both the pump and probe allows for the use of phase-sensitive lock-in detection at the difference of the two modulation frequencies.

Figure 1(b) shows the copolarized transient DT $\left(\mathrm{DT}_{\|}\right)$ spectrum obtained through a single submicron aperture at low pump powers near zero pump-probe delay $(\tau \approx 0)$. Also shown for comparison is the high-resolution cw DT spectrum, ${ }^{19}$ showing that the resonance indicated by the arrow corresponds to a single localized QD state. The linewidths in the transient spectrum are broadened by the laser bandwidth, whereas the resonances in the $\mathrm{cw}$ spectrum are homogeneously broadened. Both spectra were obtained for this figure using linearly copolarized $\Pi_{y}$ fields. Similar results were obtained using copolarized $\Pi_{x}$ fields.

Using the density matrix-formalism for a three-level system, a $\Pi_{y}$ pump creates a second order population difference, $\rho_{g g}^{(2)}(t)-\rho_{y y}^{(2)}(t)$, which decays at the relaxation rate $\Gamma_{y g}$. In this notation, $\rho_{i j}$ is the density matrix element between states $|i\rangle$ and $|j\rangle$, whereas $\Gamma_{i j}$ represents relaxation from state $|i\rangle$ to state $|j\rangle$. This population difference can be probed by either a copolarized $\Pi_{y}\left(\mathrm{DT}_{\|}\right)$or cross-polarized $\left(\mathrm{DT}_{\perp}\right) \Pi_{x}$ probe. For a $\Pi_{y}$ probe, the third-order (in the applied optical fields) induced nonlinear optical polarization field is given by $\mathbf{P}^{(3)}(t)=\left[\rho_{y g}^{(3)}(t) \boldsymbol{\mu}_{g y}+C\right.$.C. $]$. On the other hand, for a $\Pi_{x}$ probe, the induced polarization is given by $\mathbf{P}^{(3)}(t)=\left[\rho_{x g}^{(3)}(t) \boldsymbol{\mu}_{g x}+C . C\right.$. $]$. The signal field associated with either polarization is then homodyne detected with the probe. For $\mathrm{DT}_{\perp}$, a polarizer is placed in front of the detector to block the $\Pi_{y}$ polarized pump field, ensuring clean homodyne detection and zero signal for negative probe delays. In the copolarized configuration, scattering by the submicron aperture implies that at negative time the pump acts as the probe and the probe as the pump, so the negative delay signal mirrors the positive delay signal (only the positive delay signal is shown later).

In the absence of exciton spin relaxation, the crosspolarization configuration would be expected to yield about one-half the signal as the copolarized configuration, since

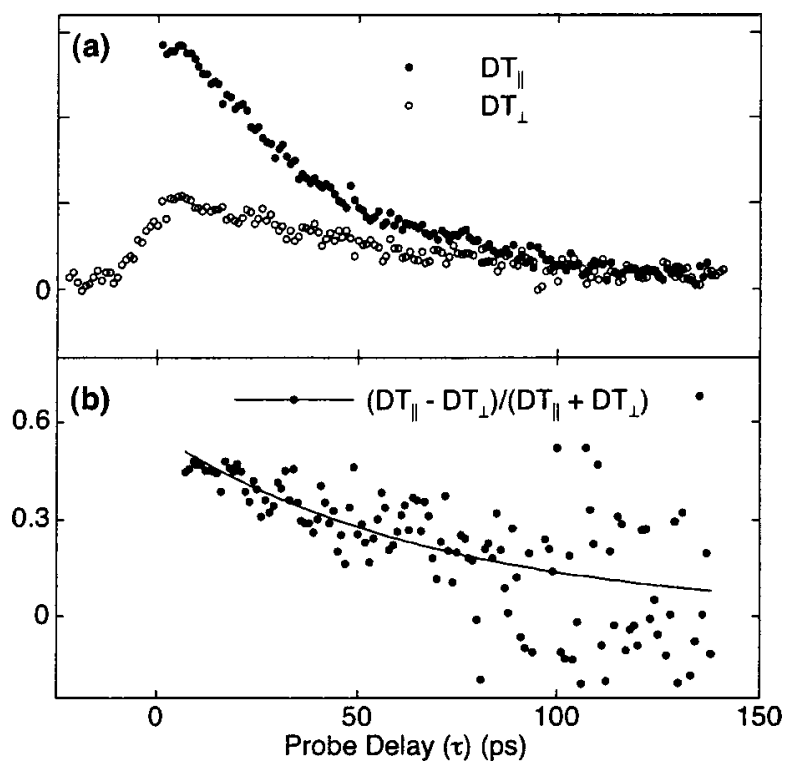

FIG. 2. (a) Copolarized ( $\mathrm{DT}_{\|}$) and cross-polarized $\left(\mathrm{DT}_{\perp}\right)$ differential transmission from the single QD state labeled by the arrow in Fig. 1. The data sets are plotted with the same vertical units. The rise of $\mathrm{DT}_{\perp}$ near $t=0$ is pulse width limited. $\mathrm{DT}_{\|}$near $t=0$ shows no such rise due to the symmetry of the copolarized detection. (b) The decay of the spin polarization. A fit to the data gives $1 / \Gamma_{y x}=140 \mathrm{ps} \pm 40 \mathrm{ps}$. Data within a pulse width of zero delay are not included in the analysis.

both $\rho_{g g}^{(2)}(t)$ and $\rho_{y y}^{(2)}(t)$ contribute to the copolarized signal but only $\rho_{g g}^{(2)}(t)$ contributes to the cross-polarized signal. This cross-polarized signal can be thought of as arising from ground state depletion, based on the $V$ system of Fig. 1(a). In addition, a lack of spin relaxation would imply that both the copolarized and cross-polarized signal would decay at the same rate, $\Gamma_{y g}$. Thus, a comparison of $\mathrm{DT}_{\|}$with $\mathrm{DT}_{\perp}$ allows for the time scale for spin relaxation within a single QD polarization doublet to be measured.

$\mathrm{DT}_{\|}$and $\mathrm{DT}_{\perp}$ are shown in Fig. 2(a) as a function of probe delay for the single QD state denoted by the arrow of Fig. 1(b). Both data sets are plotted with the same vertical units. There are two important features of this data. First, near zero delay, the magnitude of the copolarized data is about twice that of the cross-polarized data. This is consistent with the three-level model of Fig. 1(a) as discussed earlier. Second, the ratio of copolarized signal to cross-polarized signal appears to decrease as the probe delay increases. However, in the absence of spin relaxation between the two exciton states, both signals would decay at the same rate, $\Gamma_{y g}$ and the ratio would remain fixed. Thus, the data of Fig. 2(a) suggests a contribution to the dynamics that is due to spin relaxation. Also note that the decay for the copolarized data is about 40 ps.

Because the splitting is much less than $k T,(k T=600$ $\mu \mathrm{eV}$ at $T=7 \mathrm{~K})$ it is assumed that relaxation from $\left|e_{x}\right\rangle$ to $\left|e_{y}\right\rangle$ is comparable to relaxation from $\left|e_{y}\right\rangle$ to $\left|e_{x}\right\rangle$. Under the additional assumptions that the transition strengths and lifetimes for the two excitonic states are equal (which has been found experimentally to be approximately the case), ${ }^{20}$ the spin relaxation rate can be found from the ratio of the difference of the copolarized and cross-polarized DT to their sum

$\frac{\mathrm{DT}_{\|}-\mathrm{DT}_{\perp}}{\mathrm{DT}_{\|}+\mathrm{DT}_{\perp}} \propto e^{-2 \Gamma_{y x} \tau}$. 
This equation is derived under the assumption of $\delta$-function pulses and neglects so-called coherent artifacts at zero delay.

Figure 2(b) shows the difference of the copolarized and cross-polarized data shown in Fig. 2(a), divided by their sum. A fit to the data yields the spin relaxation rate, $\Gamma_{y x}$, determined for this doublet to be $1 / \Gamma_{y x}=140 \pm 40 \mathrm{ps}$. The error bars on this rate result from the error in the background (zero) DT signal and the noise in the signal itself.

The simple model of spin relaxation used for the data of Fig. 2(b) shows that the spin relaxation times are on the order of $\sim 100 \mathrm{ps}$. This is consistent with the value obtained from the opposite cross-polarization configuration, (a $\Pi_{x}$ pump and a $\Pi_{y}$ probe) in which the population $\rho_{x x}^{(2)}(t)$ and the coherence $\rho_{y g}^{(3)}(t)$ are created for a measurement of the spin relaxation rate $\Gamma_{x y}$. Spin relaxation times on the order of $\sim 100$ ps were also measured from other single QD states using this technique. For comparison, the measured lifetimes of the exciton states $\left|e_{y}\right\rangle$ and $\left|e_{x}\right\rangle\left(1 / \Gamma_{y g}\right.$ and $\left.1 / \Gamma_{x g}\right)$ are in the range of 20-40 ps.

Low temperature measurements of the spin relaxation time in thin GaAs quantum wells ( $\sim 7 \mathrm{~nm}$ wide) are on the order of tens of picoseconds, ${ }^{21,22}$ a result of strong exchange interactions in narrow quantum wells. ${ }^{5}$ However, lateral localization that results in a discrete density of states and a reduced carrier wave function overlap would be expected to inhibit spin relaxation, consistent with the measured spin relaxation times of $\sim 100 \mathrm{ps}$ reported here.

Nonlinear measurements of single interface fluctuation QDs in a magnetic field have reported a minimum spin relaxation time of $100 \mathrm{ps}$ at a field of $1.3 \mathrm{~T},{ }^{23}$ though the spin relaxation rate is expected to be strongly dependent on magnetic field; the measurements in this work are performed at zero magnetic field. Theoretical predictions of the spin relaxation time for excitons in elongated interface fluctuation QDs based on two-phonon scattering processes are on the order of several nanoseconds, ${ }^{3}$ longer than those reported here. Also, the intradoublet spin relaxation times measured here are considerably shorter than the times measured in self-assembled QDs. ${ }^{2,11,12}$ The enhanced spin relaxation in interface fluctuation QDs compared to the predictions of Takagahara et al. and to measurements in self-assembled QDs is likely related to the degree of exciton localization. In strongly localized QDs, spin scattering that relies on translational invariance or motional narrowing, such as exchange interactions or D'yakonov-Perel mechanisms, is strongly suppressed. However, photoluminescence imaging studies of narrow GaAs/ $\mathrm{AlGaAs}$ quantum wells find broad resonance features (degenerate with sharp photoluminescence lines) that emerge with an increase in power, and show data that suggests the presence of delocalized or weakly localized excitons. ${ }^{24}$ Also, biexponential photon echo decays attributable to a class of weakly localized excitons nearly degenerate with more strongly localized excitons have been observed in similar disordered quantum wells. ${ }^{25}$ Weak localization therefore implies that the motional-narrowing type mechanisms responsible for fast spin relaxation in quantum wells may not be entirely suppressed in growth-interrupted quantum wells of the type studied here.

In summary, excitons confined to single quantum dots have been studied through copolarized and cross-polarized transient differential transmission spectroscopy. Comparison of the copolarized to cross-polarized DT leads to a direct measurement of the spin relaxation rate between heavy-hole exciton polarization eigenstates. The measured relaxation times are on the order of $\sim 100 \mathrm{ps}$, longer than the time observed in high quality quantum wells, but considerably shorter than either the times predicted for interface fluctuation QDs or measured for self-assembled QDs. The presence of weakly localized excitonic states in growth-interrupted quantum wells could contribute to an enhanced spin relaxation rate.

This work was supported in part by the the Office of Naval Research, the National Security Agency (NSA) and Advanced Research and Development Activity (ARDA) under Army Research Office (ARO) Contract No. DAAG5598-1-0373, the Air Force Office of Scientific Research under Grant No. F49620-99-1-0045, NSF-FOCUS, and DARPA/ Spins.

${ }^{1}$ Optical Orientation, Modern Problems in Condensed Matter Sciences Vol. 8, edited by F. Meier and B. Zakharchenya (North-Holland, Amsterdam, 1984).

${ }^{2}$ H. Gotoh, H. Ando, H. Kamada, A. Chavez-Pirson, and J. Temmyo, Appl. Phys. Lett. 72, 1341 (1998).

${ }^{3}$ T. Takagahara, J. Lumin. 87, 308 (2000).

${ }^{4}$ M. I. D'yakonov and V. I. Perel, Zh. Eksp. Teor. Fiz. 60, 1954 (1971) [Sov. Phys. JETP 33, 108 (1971)].

${ }^{5}$ M. Maialle, E. de Andrada e Silva, and L. Sham, Phys. Rev. B 47, 15776 (1993).

${ }^{6}$ A. Imamoğlu, D. D. Awschalom, G. Burkard, D. P. DiVincenzo, D. Loss, M. Sherwin, and A. Small, Phys. Rev. Lett. 83, 4204 (1999).

${ }^{7}$ D. P. DiVincenzo, J. Appl. Phys. 85, 4785 (1999).

${ }^{8}$ D. Loss and D. P. DiVincenzo, Phys. Rev. A 57, 120 (1998).

${ }^{9}$ P. Chen, C. Piermarocchi, and L. J. Sham, Phys. Rev. Lett. 87, 067401 (2001).

${ }^{10}$ Y. Ohno, D. K. Young, B. Beschoten, F. Matsukura, H. Ohno, and D. D. Awschalom, Nature (London) 402, 790 (1999).

${ }^{11}$ M. Paillard, X. Marie, P. Renucci, T. Amand, A. Jbeli, and J. M. Gérard, Phys. Rev. Lett. 86, 1634 (2001).

${ }^{12}$ A. S. Lenihan, M. V. G. Dutt, D. G. Steel, S. Ghosh, and P. K. Bhattacharya, Phys. Rev. Lett. 88, 223601 (2002).

${ }^{13}$ T. H. Stievater, X. Li, D. G. Steel, D. Gammon, D. Park, and D. S. Katzer, Phys. Rev. B 65, 205319 (2002).

${ }^{14}$ T. H. Stievater, X. Li, D. G. Steel, D. S. Katzer, D. Park, D. Gammon, L. J. Sham, and C. Piermarocchi, Phys. Rev. Lett. 87, 133603 (2001).

${ }^{15}$ D. Gammon, E. S. Snow, and D. S. Katzer, Appl. Phys. Lett. 67, 2391 (1995).

${ }^{16}$ D. Gammon, E. S. Snow, B. V. Shanabrook, D. S. Katzer, and D. Park, Science 273, 87 (1996).

${ }^{17}$ D. Gammon, E. S. Snow, B. V. Shanabrook, D. S. Katzer, and D. Park, Phys. Rev. Lett. 76, 3005 (1996).

${ }^{18}$ G. Chen, T. H. Stievater, E. T. Batteh, X. Li, D. G. Steel, D. Gammon, D. S. Katzer, D. Park, and L. J. Sham, Phys. Rev. Lett. 88, 117901 (2002).

${ }^{19}$ N. H. Bonadeo, G. Chen, D. Gammon, D. S. Katzer, D. Park, and D. G. Steel, Phys. Rev. Lett. 81, 2759 (1998).

${ }^{20}$ J. R. Guest, T. H. Stievater, X. Li, J. Cheng, D. G. Steel, D. S. Katzer, D. Park, D. Gammon, C. Ell, A. Thränhardt, H. M. Gibbs, and G. Khitrova, Phys. Rev. B 65, 241310 (2002).

${ }^{21}$ T. C. Damen, K. Leo, J. Shah, and J. E. Cunningham, Appl. Phys. Lett. 58, 1902 (1991)

${ }^{22}$ A. Malinowski, R. S. Britton, T. Grevatt, R. T. Harley, D. A. Ritchie, and M. Y. Simmons, Phys. Rev. B 62, 13034 (2000).

${ }^{23}$ G. Chen, N. H. Bonadeo, D. G. Steel, D. Gammon, D. S. Katzer, D. Park, and L. J. Sham, Science 289, 1906 (2000).

${ }^{24}$ Q. Wu, R. D. Grober, D. Gammon, and D. S. Katzer, Phys. Rev. Lett. 83, 2652 (1999).

${ }^{25}$ J. Erland, J. C. Kim, N. H. Bonadeo, D. Gammon, D. S. Katzer, and D. G. Steel, Phys. Rev. B 60, R8497 (1999). 